\title{
Interval-valued belief entropies for Dempster-Shafer structures
}

\author{
Yige Xue ${ }^{1}$ Yong Deng ${ }^{1,2,3}$ \\ Accepted: 20 May 2021 / Published online: 4 June 2021 \\ (c) The Author(s), under exclusive licence to Springer-Verlag GmbH Germany, part of Springer Nature 2021
}

\begin{abstract}
In practical application problems, the uncertainty of an unknown object is often very difficult to accurately determine, so Yager proposed the interval-valued entropies for Dempster-Shafer structures, which is based on Dempster-Shafer structures and classic Shannon entropy and is an interval entropy model. Based on Dempster-Shafer structures and classic Shannon entropy, the interval uncertainty of an unknown object is determined, which provides reference for theoretical research and provides help for industrial applications. Although the interval-valued entropies for Dempster-Shafer structures can solve the uncertainty interval of an object very efficiently, its application scope is only a traditional probability space. How to extend it to the evidential environment is still an open issue. This paper proposes interval-valued belief entropies for Dempster-Shafer structures, which is an extension of the interval-valued entropies for Dempster-Shafer structures. When the belief entropy degenerates to the classic Shannon entropy, the interval-valued belief entropies for Dempster-Shafer structures will degenerate into the interval-valued entropies for Dempster-Shafer structures. Numerical examples are applied to verify the validity of the interval-valued belief entropies for Dempster-Shafer structures. The experimental results demonstrate that the proposed entropy can obtain the interval uncertainty value of a given uncertain object successfully and make decision effectively.
\end{abstract}

Keywords Dempster-Shafer structures · Belief entropy · Uncertainty · Shannon entropy $\cdot$ Interval-valued entropies

\section{Introduction}

There are a lot of uncertainties in the real world (Potyka et al. 2015; Atanassov 1999) and plenty of issues about uncertainty (Biró et al. 2019; Telcs et al. 2019). In order to deal with the uncertainties (Karci 2018; Abellan and Bosse 2018), many mathematical theories are proposed, such as fuzzy sets (Xue et al. 2020) and entropy (Tuğal and Karc1 2019). Beer et al. (2020) proposed the case of permutation-invariant criteria. Cao et al. (2019) proposed the multichannel EEG recordings under some uncertain environment. Du et al. (2019) proposed an improved Dempster-Shafer evidence theory under gray relational analysis. Jiang et al. (2019) proposed a novel probability transformation model based on a cor-

Yong Deng

dengentropy@uestc.edu.cn

1 Institute of Fundamental and Frontier Sciences, University of Electronic Science and Technology of China, Chengdu 610054, China

2 School of Education, Shaanxi Normal University, Xi'an 710062, China

3 School of Knowledge Science, Japan Advanced Institute of Science and Technology, Nomi, Ishikawa 923-1211, Japan relation coefficient of belief functions. Zhou et al. (2018) proposed a new multisensor data fusion method under modified Dempster-Shafer evidence theory. Yager et al. (2019) applied the fuzzy sets to linguistic data science by drawing on the iPad. Khan and Anwar (2019) proposed a new timedomain data fusion method by using weighted evidence and Dempster-Shafer combination rule and applied it to classify object. Kreinovich et al. (2021) studied how to reconcile maximum entropy approach with intuition. Jirousek and Shenoy (2018) proposed a novel entropy of belief functions in the Dempster-Shafer theory. Yager (2019) extended set measures to Pythagorean fuzzy sets.

Among these theories and models, a new belief entropy (Deng 2020), named as Deng entropy, is proposed to measure the uncertainty of mass function (Wang and Xiao 2019). The belief entropy is an extend of information entropy, which can evaluate uncertainties more flexible than information entropy (Huang et al. 2019). The belief entropy is based on the evidence theory, which means that the belief entropy can represent the uncertainties under the frame of discernment effectively (Mambé et al. 2018). Relying on the advantages on indicating vague information, the belief entropy has been widely studied by scholars at home and abroad (Liu et al. 
2019). Some scholars has applied the belief entropy to deal with decision-making problems (Xiao 2019; Huang et al. 2019). Xiao (2019) proposed the evidential fuzzy multicriteria decision making based on belief entropy. Fan et al. (2020) proposed multisensor fusion method based on the belief entropy under evidential environment. Ozkan (2018) compared Shannon entropy with Deng entropy and improved Deng entropy for measuring biodiversity under an uncertain environment. Abellán (2017) has analyzed the properties of Deng entropy.

Recently, Yager proposed the interval-valued entropies for Dempster-Shafer structures (Yager 2018), which is based on Dempster-Shafer structure and has promising aspects (Deng and Jiang 2019). Dempster-Shafer structure is a mapping from the focal element to the interval $[0,1]$ in a given space. It has a strong ability to represent unknown information. Through the focal elements of a given space, the possibility and certainty of each focal element can be obtained. The belief and plausibility of each focal element can be obtained through the possibility and certainty of each focal element and the Dempster-Shafer structure. The belief and plausibility of each focal element can determine the confidence interval of the corresponding focal element, which means that the Dempster-Shafer structure can express unknown information very efficiently. However, what is the corresponding interval-valued entropies for Dempster-Shafer structures under belief entropy is still an open issue to be addressed.

This paper proposes the interval-valued belief entropies for Dempster-Shafer structures, which is an interval-valued entropy. The proposed entropy is based on Dempster-Shafer structure and belief entropy, which is an efficient way to measure the uncertainty of location information. Through the focal elements in a given space, the proposed entropy can obtain the possibility and certainty of each focal element. Furthermore, the belief and plausibility of each focal element can be obtained. Based on the belief and plausibility and cardinality of each focal element, the proposed entropy can be obtained. The proposed entropy is based on the interval-valued entropies for Dempster-Shafer structures. When the belief entropy degenerates into the classic Shannon entropy, the proposed entropy will degenerate into the interval-valued entropies for Dempster-Shafer structures. And through a comparative experiment, we verified that the proposed entropy can obtain more information about an unknown object. At the same time, because the proposed entropy is an interval entropy model, it is more in line with human intuition, which provides a basis for practical applications.

The remaining of this paper is structured as follows. Section 2 introduces the preliminary. Section 3 presents the interval-valued belief entropies for Dempster-Shafer structures. Section 4 illustrates the flexibility and accuracy of the interval-valued belief entropies for Dempster-Shafer structures. Section 5 summarizes the whole paper.

\section{Preliminaries}

The world is full of unknowns, and the unknown world will have many unknown problems (Wang et al. 2019; Zhang and Deng 2021). Unknown information needs to be represented by tools that represent information (Turhan and Demirekler 2017; Wang et al. 2019). After the unknown information is expressed, many unknown means are needed to process it (Kern-Isberner and Rödder 2004; Pan and Deng 2020).

\subsection{Dempster-Shafer structures}

The real world is very uncertain and complex (Yager 1999; Zhang et al. 2017). Addressing uncertainty is an open issue (Prajapati and Saha 2019). Evidence theory is widely applied and generalized (Seiti and Hafezalkotob 2018; Jaunzemis et al. 2019). Dempster-Shafer structure is a very efficient tool for representing unknown information (Zhang et al. 2019). The performance of Dempster-Shafer structure is very powerful (Chao et al. 2019). Dempster-Shafer structure maps each non-empty crisp subset of a given space to a value in the [0,1] space (Vandoni et al. 2019). Through the DempsterShafer structure, we can get the belief and plausibility of each non-empty crisp subset in a given space (Jamal et al. 2019; Ferson et al. 2015). The belief and plausibility of each nonempty crisp subset in a given space can form an interval (Hurley et al. 2019; Zhang et al. 2019). This interval value is an important indicator for evaluating the reliability of a nonempty crisp subset (Wang 2018; Kuzemsky 2018). Given a space $X$ consists of a collection $F=\left\{F_{1}, \ldots, F_{q}\right\}$ of nonempty crisp subsets of $X$, the Dempster-Shafer structure is a mapping $m\left(F_{j}\right) \in[0,1]$ satisfying the following conditions:

Definition 1 (Dempster-Shafer structure) (Shafer 1976)

$$
\begin{aligned}
& m\left(F_{j}\right)>0 \\
& \sum_{j=1}^{q} m\left(F_{j}\right)=1
\end{aligned}
$$

Two important measures that can be associated with a Dempster-Shafer structure $m$ are the measures of plausibility and belief, $\mathrm{Pl}$ and $\mathrm{Bel}$, as follows:

$$
\begin{aligned}
& \operatorname{Pl}(A)=\sum_{j, A \cap F_{j} \neq \emptyset} m\left(F_{j}\right) \\
& \operatorname{Bel}(A)=\sum_{j, F_{j} \subseteq A} m\left(F_{j}\right)
\end{aligned}
$$


Assume $A$ is a crisp subset of $X$, then the certainty and possibility of $A$ is defined as follows:

$$
\begin{aligned}
& \operatorname{Pl}(A)=\sum_{j=1}^{q} \operatorname{Poss}\left(A / F_{j}\right) m\left(F_{j}\right) \\
& \operatorname{Bel}(A)=\sum_{j=1}^{q} \operatorname{Cert}\left(A / F_{j}\right) m\left(F_{j}\right)
\end{aligned}
$$

where,

$$
\begin{aligned}
& \operatorname{Poss}\left(A / F_{j}\right)=1 \text { if } A \cap F_{j} \neq \emptyset \\
& \operatorname{Poss}\left(A / F_{j}\right)=0 \text { if } A \cap F_{j}=\emptyset \\
& \operatorname{Cert}\left(A / F_{j}\right)=1 \text { if } F_{j} \subseteq A \\
& \operatorname{Cert}\left(A / F_{j}\right)=0 \text { if } F_{j} \not \subseteq A
\end{aligned}
$$

\subsection{Interval-valued entropies for Dempster-Shafer structures}

The interval-valued entropies for Dempster-Shafer structures proposed by Yager include Shannon-like intervalvalued entropies and Gini entropy of Dempster-Shafer belief structure. Because Shannon entropy is the most classic entropy, the interval-valued entropies for Dempster-Shafer structures mentioned in this article are Shannon-like intervalvalued entropies.

Assume $m$ is a Dempster-Shafer structure on $X=$ $\left\{x_{1}, \ldots, x_{n}\right\}$ with focal elements $G=\left\{G_{1}, \ldots, G_{q}\right\}$ and $m\left(G_{j}\right)=\alpha_{j}$. where $\alpha_{j} \in[0,1]$ and $\sum_{j=1}^{q} \alpha_{j}=1$. The definition of Shannon-like interval-valued entropies is as follows:

Definition 2 (Shannon-like interval-valued entropies)(Yager 2018)

$S(m) \in\left[S_{L}(m), S_{U}(m)\right]$

where,

$$
\begin{aligned}
& S_{\mathrm{L}}(m)=-\sum_{G_{j} \in G} m\left(G_{j}\right) \log _{2}\left(\mathrm{Pl}_{m}\left(G_{j}\right)\right) \\
& S_{\mathrm{U}}(m)=-\sum_{G_{j} \in G} m\left(G_{j}\right) \log _{2}\left(\operatorname{Bel}_{m}\left(G_{j}\right)\right)
\end{aligned}
$$

where $P l_{\mathrm{m}}(A)$ and $\mathrm{Bel}_{\mathrm{m}}(A)$ indicate the plausibility and belief of $A$ with respect to the Dempster-Shafer structure $m$, respectively.

\subsection{Tsallis entropy}

The definition of Tsallis entropy under a probability distribution $p_{i}$ is as follows:
Definition 3 (Tsallis entropy) (Tsallis 1988)

$$
S_{q}=k \frac{1-\sum_{i=1}^{W} p_{i}^{q}}{q-1} \quad q \in R
$$

When $k$ is a conventional positive constant and $\sum_{i=1}^{W} p_{i}=$ 1 , it is obvious that $\lim _{q \rightarrow 1} S_{q}=-k \sum_{i=1}^{W} p_{i} \ln p_{i}$. In this way, the Tsallis entropy is degenerated as classical information entropy.

\subsection{Belief entropy}

Given $n$ is a mass function under frame of discernment $Y$, the definition of belief entropy is as follows:

Definition 4 (Belief entropy) (Deng 2020)

$E_{\mathrm{d}}=-\sum_{C \subseteq Y} n(C) \log \frac{n(C)}{2^{|C|}-1}$

where $|C|$ is the cardinality of $C$.

Belief entropy is the generalization of Shannon entropy (Mambe et al. 2018; Khoshtinat et al. 2019). When the mass function is degenerated as a probability distribution, belief entropy is degenerated as Shannon entropy.

\subsection{Golden rule method}

Assume $E$ is the any focal element of $\Theta . P l(E)$ and $B e l(E)$ are the plausibility and belief of $E$, respectively. The definition of $E$ is as follows:

Definition 5 (Golden Rule Method) (Yager 2017)

$\operatorname{Rep}(E)=m+(0.5-m) r$

where,

$$
\begin{aligned}
& m=(P l(E)+B e l(E)) / 2 \\
& r=P l(E)-B e l(E)
\end{aligned}
$$

\section{Interval-valued belief entropies for Dempster-Shafer structures}

The unknown world is tens of thousands (Deng 2020; Deng and Deng 2021), and people cannot accurately judge the uncertainty of an unknown information (Pan et al. 2019; Jiang et al. 2019). But we can give an interval of the uncertainty of an object and then control the uncertainty of an object within a range, which is also a way of expressing unknown information. On this basis, it can help people deal with unknown information. Yager proposed the interval-valued entropies 
for Dempster-Shafer structures, which is an interval entropy model based on Dempster-Shafer structures and classic information entropy. Although the interval-valued entropies for Dempster-Shafer structures can solve the uncertainty interval of an object very efficiently, its scope of application is only a traditional probability space. If extended to the evidence environment, this is the interval value proposed in this article.

In this section, an interval-valued belief entropy for Dempster-Shafer structures is presented. Assume $m$ is a Dempster-Shafer structure on $X=\left\{x_{1}, \ldots, x_{n}\right\}$ with focal elements $G=\left\{G_{1}, \ldots, G_{q}\right\}$ and $m\left(G_{j}\right)=\alpha_{j}$. where $\alpha_{j} \in[0,1]$ and $\sum_{j=1}^{q} \alpha_{j}=1$. The definition of the intervalvalued belief entropy for Dempster-Shafer structures is as follows:

Definition 6 (Interval-valued belief entropies for DempsterShafer structures)

$S(m)=\left[S_{L}(m), S_{U}(m)\right]$

where

$$
\begin{aligned}
& S_{L}(m)=-\sum_{G_{j} \in G} m\left(G_{j}\right) \log _{2} \frac{\mathrm{Pl}_{m}\left(G_{j}\right)}{2^{\left|G_{j}\right|}-1} \\
& S_{H}(m)=-\sum_{G_{j} \in G} m\left(G_{j}\right) \log _{2} \frac{\mathrm{Bel}_{m}\left(G_{j}\right)}{2^{\left|G_{j}\right|}-1}
\end{aligned}
$$

where

$$
\begin{aligned}
& \operatorname{Pl}_{m}\left(G_{j}\right)=\sum_{k=1}^{q} \operatorname{Poss}\left(G_{j} / G_{k}\right) m\left(G_{k}\right) \\
& \operatorname{Bel}_{m}\left(G_{j}\right)=\sum_{k=1}^{q} \operatorname{Cert}\left(G_{j} / G_{k}\right) m\left(G_{k}\right)
\end{aligned}
$$

Hence,

$$
\begin{aligned}
& S_{L}(m)=-\sum_{j=1}^{q} m\left(G_{j}\right) \log _{2} \frac{\sum_{k=1}^{q} \operatorname{Poss}\left(G_{j} / G_{k}\right) m\left(G_{k}\right)}{2^{\left|G_{j}\right|}-1} \\
& S_{U}(m)=-\sum_{j=1}^{q} m\left(G_{j}\right) \log _{2} \frac{\sum_{k=1}^{q} \operatorname{Cert}\left(G_{j} / G_{k}\right) m\left(G_{k}\right)}{2^{\left|G_{j}\right|}-1}
\end{aligned}
$$

The flowchart of the proposed entropy is shown in Fig. 1.

Theorem 1 When the belief entropy degenerates into the classic Shannon entropy, the interval-valued belief entropies for Dempster-Shafer structures will degenerate into the interval-valued entropies for Dempster-Shafer structures.
Proof Relying on Eqs. $(20,21)$, we have the equations as follows:

$$
\begin{aligned}
& S_{\mathrm{L}}(m)=-\sum_{G_{j} \in G} m\left(G_{j}\right) \log _{2} \frac{P l_{m}\left(G_{j}\right)}{2^{\left|G_{j}\right|}-1} \\
& S_{\mathrm{H}}(m)=-\sum_{G_{j} \in G} m\left(G_{j}\right) \log _{2} \frac{B e l_{m}\left(G_{j}\right)}{2^{\left|G_{j}\right|}-1}
\end{aligned}
$$

When the belief entropy degenerates into the classic Shannon entropy, we can obtain the following equations:

$$
\begin{aligned}
& -\sum_{G_{j} \in G} m\left(G_{j}\right) \log _{2} \frac{P l_{m}\left(G_{j}\right)}{2^{\left|G_{j}\right|}-1} \\
& =-\sum_{G_{j} \in G} m\left(G_{j}\right) \log _{2} P l_{m}\left(G_{j}\right) \\
& -\sum_{G_{j} \in G} m\left(G_{j}\right) \log _{2} \frac{B e l_{m}\left(G_{j}\right)}{2^{\left|G_{j}\right|}-1} \\
& =-\sum_{G_{j} \in G} m\left(G_{j}\right) \log _{2} \operatorname{Bel}_{m}\left(G_{j}\right)
\end{aligned}
$$

The interval-valued belief entropies for Dempster-Shafer structures will degenerate into the interval-valued entropies for Dempster-Shafer structures.

Then, the interval-valued belief entropies for DempsterShafer structures will degenerate into the interval-valued entropies for Dempster-Shafer structures.

Theorem 2 Assume $m$ is a Bayesian Dempster-Shafer structure with $F_{i}=\left\{x_{i}\right\}$ and $m\left(F_{i}\right)=p_{i}$ for $i=1$ to $n$. For an interval-valued belief entropy for Dempster-Shafer structure, $S(m)=\left[S_{L}(m), S_{U}(m)\right]$, on $m$, then $S_{L}(m)=S_{U}(m)$.

Proof Relying on the equations of Eqs. $(24,25)$, we have the equations as follows:

$$
\begin{aligned}
& S_{\mathrm{L}}(m)=-\sum_{j=1}^{q} m\left(G_{j}\right) \log \frac{\sum_{k=1}^{q} \operatorname{Poss}\left(G_{j} / G_{k}\right) m\left(G_{k}\right)}{2^{\left|G_{j}\right|}-1} \\
& S_{\mathrm{U}}(m)=-\sum_{j=1}^{q} m\left(G_{j}\right) \log \frac{\sum_{k=1}^{q} \operatorname{Cert}\left(G_{j} / G_{k}\right) m\left(G_{k}\right)}{2^{\left|G_{j}\right|}-1}
\end{aligned}
$$

Since $m$ is a Bayesian Dempster-Shafer structure, then $\operatorname{Cert}\left(G_{i} / G_{k}\right)=\operatorname{Poss}\left(G_{i} / G_{k}\right)$. 
Fig. 1 The flowchart of the proposed entropy

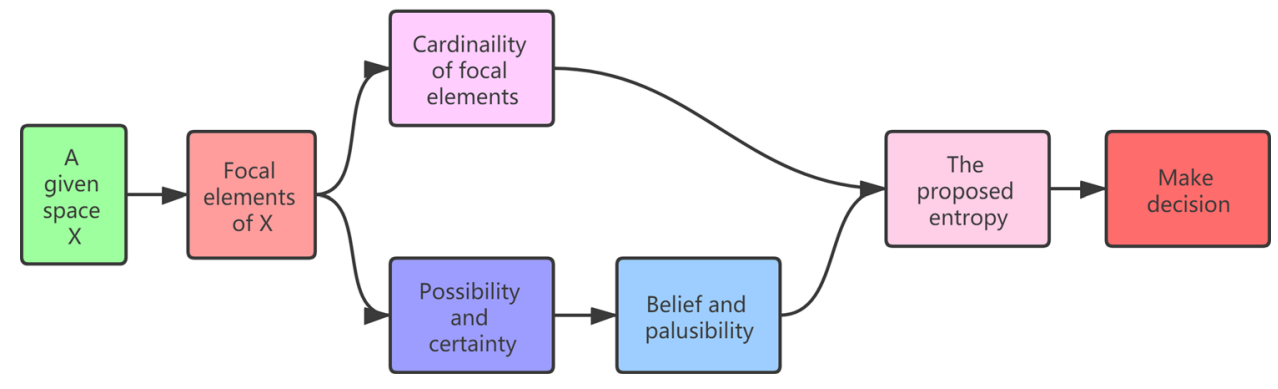

$$
\begin{aligned}
S_{\mathrm{L}}(m) & =-\sum_{i=1}^{q} m\left(G_{i}\right) \log \frac{\sum_{k=1}^{q} \operatorname{Poss}\left(G_{i} / G_{k}\right) m\left(G_{k}\right)}{2^{\left|G_{i}\right|}-1} \\
& =-\sum_{i=1}^{n} m\left(G_{i}\right) \log \sum_{k=1}^{n} \operatorname{Poss}\left(G_{i} / G_{k}\right) m\left(G_{k}\right) \\
& =-\sum_{i=1}^{n} m\left(G_{i}\right) \log m\left(G_{i}\right)=-\sum_{i=1}^{n} p_{i} \log p_{i} \\
S_{\mathrm{U}}(m) & =-\sum_{i=1}^{q} m\left(G_{i}\right) \log \frac{\sum_{k=1}^{q} \operatorname{Cert}\left(G_{i} / G_{k}\right) m\left(G_{k}\right)}{2^{\left|G_{i}\right|}-1} \\
& =-\sum_{i=1}^{n} m\left(G_{i}\right) \log \sum_{k=1}^{n} \operatorname{Cert}\left(G_{i} / G_{k}\right) m\left(G_{k}\right) \\
& =-\sum_{i=1}^{n} m\left(G_{i}\right) \log m\left(G_{i}\right)=-\sum_{i=1}^{n} p_{i} \log p_{i}
\end{aligned}
$$

So, we come to the conclusion that $S_{L}(m)=S_{U}(m)$.

Example 1 Assume Dempster-Shafer structure $m$ on the focal elements $F=\left\{F_{1}, F_{2}, F_{3}\right\}$ of a given space $X=$ $\left\{x_{1}, x_{2}\right\}$ with where $F_{1}=\left\{x_{1}\right\}$ with $m\left(F_{1}\right)=0.1, F_{2}=\left\{x_{2}\right\}$ with $m\left(F_{2}\right)=0.1$ and $F_{3}=\left\{x_{1}, x_{2}\right\}$ with $m\left(F_{3}\right)=0.8$.

Relying on Eqs. $(24,25)$, we have the equations as follows:

$$
\begin{aligned}
S_{\mathrm{Y}}(m)= & -0.1 \times \log _{2} \frac{P l_{m}\left(F_{1}\right)}{2^{|1|}-1}-0.1 \times \log _{2} \frac{P l_{m}\left(F_{2}\right)}{2^{|1|}-1} \\
& -0.8 \times \log _{2} \frac{P l_{m}\left(F_{3}\right)}{2^{|2|}-1} \\
= & -0.1 \times \log _{2} \frac{0.1+0.8}{2^{|1|}-1}-0.1 \times \log _{2} \frac{0.1+0.8}{2^{|1|}-1} \\
& -0.8 \times \log _{2} \frac{0.1+0.1+0.8}{2^{|2|}-1} \\
= & 1.298 \\
S_{\mathrm{H}}(m)= & -0.1 \times \log _{2} \frac{\mathrm{Bel}_{m}\left(F_{1}\right)}{2^{|1|}-1}-0.1 \times \log _{2} \frac{\mathrm{Bel}_{m}\left(F_{2}\right)}{2^{|1|}-1} \\
& -0.8 \times \log _{2} \frac{\mathrm{Bel}_{m}\left(F_{3}\right)}{2^{|2|}-1} \\
= & -0.1 \times \log _{2} \frac{0.1}{2^{|1|}-1}-0.1 \times \log _{2} \frac{0.1}{2^{|1|}-1}
\end{aligned}
$$

$$
\begin{aligned}
& -0.8 \times \log _{2} \frac{0.1+0.1+0.8}{2^{|2|}-1} \\
= & 1.932
\end{aligned}
$$

Hence, we see that

$S(m)=[1.298,1.932]$

Here, [1.298, 1.932] is the interval-valued belief entropy for Dempster-Shafer structure on $X$. Through this example, we know that the proposed entropy can successfully calculate the belief interval of an unknown object.

Example 2 Assume Dempster-Shafer structure $m$ on $X=$ $\left\{x_{1}, x_{2}\right\}$ with focal elements $F=\left\{F_{1}, F_{2}\right\}$ where $F_{1}=\left\{x_{1}\right\}$ with $m\left(F_{1}\right)=0.5$ and $F_{2}=\left\{x_{2}\right\}$ with $m\left(F_{2}\right)=0.5$.

Relying on the equations of Eqs. $(24,25)$, we have the equations as follows:

$$
\begin{aligned}
S_{\mathrm{L}}(m) & =-0.5 \times \log _{2} \frac{\mathrm{Pl}_{m}\left(F_{1}\right)}{2^{|1|}}-1-0.5 \times \log _{2} \frac{P l_{m}\left(F_{2}\right)}{2^{|1|}-1} \\
& =-0.5 \times \log _{2} \frac{0.5}{2^{|1|}-1}-0.5 \times \log _{2} \frac{0.5}{2^{|1|}-1}=1 \\
S_{\mathrm{U}}(m) & =-0.5 \times \log _{2} \frac{\mathrm{Bel}_{m}\left(F_{1}\right)}{2^{|1|}-1}-0.5 \times \log _{2} \frac{\operatorname{Bel}_{m}\left(F_{2}\right)}{2^{|1|}-1} \\
& =-0.5 \times \log _{2} \frac{0.5}{2^{|1|}-1}-0.5 \times \log _{2} \frac{0.5}{2^{|1|}-1}=1
\end{aligned}
$$

Hence, we see that

$S(m)=[1,1]=1$

Here, $[1,1]$ is the interval-valued belief entropy for Dempster-Shafer structure on $X$. In this example, $m$ is a Bayesian Dempster-Shafer structure. From the calculation results, the upper and lower limits of the interval entropy that can be calculated by the proposed entropy are the same. That is, the proposed entropy can calculate the uncertainty of an unknown object accurately.

Example 3 Assume Dempster-Shafer structure $m$ on the focal elements $F=\left\{F_{1}, F_{2}\right\}$ of a given space $X=$ $\left\{x_{1}, x_{2}, x_{3}\right\}$ where $F_{1}=\left\{x_{1}\right\}$ with $m\left(F_{1}\right)=0.6, F_{2}=$ $\left\{x_{2}, x_{3}\right\}$ with $m\left(F_{2}\right)=0.4$. 
Relying on the equations of Eqs. $(24,25)$, we have the equations as follows:

$$
\begin{aligned}
S_{\mathrm{Y}}(m) & =-0.6 \times \log _{2} \frac{\mathrm{Pl}_{m}\left(F_{1}\right)}{2^{|1|}-1}-0.4 \times \log _{2} \frac{\mathrm{Pl}_{m}\left(F_{2}\right)}{2^{|2|}-1} \\
& =-0.6 \times \log _{2} \frac{0.6}{2^{|1|}-1}-0.4 \times \log _{2} \frac{0.4}{2^{|2|}-1} \\
& =1.605 \\
S_{\mathrm{H}}(m) & =-0.6 \times \log _{2} \frac{\mathrm{Bel}_{m}\left(F_{1}\right)}{2^{|1|}-1}-0.4 \times \log _{2} \frac{\mathrm{Bel}_{m}\left(F_{2}\right)}{2^{|2|}-1} \\
& =-0.6 \times \log _{2} \frac{0.6}{2^{|1|}-1}-0.4 \times \log _{2} \frac{0.4}{2^{|2|}-1} \\
& =1.605
\end{aligned}
$$

Hence, we see that

$S(m)=[1.605,1.605]$

Here, $[1.605,1.605]$ is the interval-valued belief entropy for Dempster-Shafer structure on $X$. In this example, the focal elements do not want to communicate with each other, indicating that this example is a special case. Through this example, we know that the proposed entropy can successfully calculate the belief interval of an unknown object under special case.

Example 4 A person is walking at a crossroads, and there are three roads to choose by the person. It means that three possible choices $A_{i}(i=1,2,3)$ can be chosen by the person and three attributes $y_{1}$ (length), $y_{2}$ (brightness) and $y_{3}$ (humidity) are taken into account in the issue. From a traveler's perspective, they tend to choose the path with the least uncertainty. According to the properties of belief entropy, the belief entropy can represent uncertainty effectively.

Assume the Dempster-Shafer structures $m_{1}, m_{2}$ and $m_{3}$ based on the space $Y=\left\{y_{1}, y_{2}, y_{3}\right\}$ represent alternatives $A_{1}, A_{2}$ and $A_{3}$, respectively. According to experts, the alternative $A_{3}$ is the best choice.

Assume Dempster-Shafer structures $m_{1}, m_{2}$ and $m_{3}$ on the focal elements $F_{1}=\left\{F_{11}, F_{12}, F_{13}\right\}, F_{2}=\left\{F_{21}\right\}$ and $F_{3}=\left\{F_{31}, F_{32}, F_{33}\right\}$ of a given space $Y=\left\{y_{1}, y_{2}, y_{3}\right\}$, respectively, such that $F_{11}=\left\{y_{1}\right\}, F_{12}=\left\{y_{2}\right\}, F_{13}=\left\{y_{3}\right\}$, $F_{21}=\{Y\}, F_{31}=\left\{y_{1}, y_{2}\right\}, F_{32}=\left\{y_{1}, y_{3}\right\}$ and $F_{33}=$ $\left\{y_{2}, y_{3}\right\}$.

The Dempster-Shafer structures $m_{1}, m_{2}$ and $m_{3}$ are as follows:

$$
\begin{aligned}
& m_{1}\left(\left\{y_{1}\right\}\right)=1 / 3, m_{1}\left(\left\{y_{2}\right\}\right)=1 / 3, m_{1}\left(\left\{y_{3}\right\}\right)=1 / 3 \\
& m_{2}(\{Y\})=1 \\
& m_{3}\left(\left\{y_{1}, y_{2}\right\}\right)=0.2, m_{3}\left(\left\{y_{1}, y_{3}\right\}\right)=0.4, \\
& m_{3}\left(\left\{y_{2}, y_{3}\right\}\right)=0.4
\end{aligned}
$$

Relying on the equations of Eqs. $(24,25)$, we can obtain Table 1 as follows:

Hence, we can obtain the interval values of the proposed entropy of $m_{1}, m_{2}$ and $m_{3}$ in Table 2:

According to Eq. (16), we can obtain the golden rule method values of the proposed entropy of $A_{1}, A_{2}$ and $A_{3}$ in Table 3:

From the discussion above, one get that $\operatorname{Rep}\left(A_{2}\right)>$ $\operatorname{Rep}\left(A_{1}\right)>\operatorname{Rep}\left(A_{3}\right)$. Then, we can find the choice $A_{3}$ is the best choice in this problem, which is consistent with the experts' judgment. It means that the proposed algorithm is validity in issue of decision making. This shows that the proposed entropy can successfully solve practical problems in life.

\section{Application}

The world is full of unknown information, and the uncertainty measurement of location information has always been an important issue of information fusion (Kang et al. 2019; Deng and Jiang 2019). Since the end of 2019, the new crown virus has raged, and the global economy and society are all in crisis. In order to effectively prevent infection by the new coronavirus, suppose we now have a vaccine, denoted as B. Before the vaccine is marketed, we need to use the relevant uncertainty measurement model to measure the vaccine. If the uncertainty of the vaccine is too large, then the vaccine is not suitable for injections to the relevant population, because we do not know whether the vaccine will eventually have a positive or negative impact on the person being injected.

Table 1 The upper and lower limits of the proposed entropy of $m_{1}, m_{2}$ and $m_{3}$

\begin{tabular}{llll}
\hline Alternatives & $m_{1}$ & $m_{2}$ & $m_{3}$ \\
\hline$S_{\mathrm{L}}\left(m_{\mathrm{i}}\right)$ & $\log _{2} 3$ & $\log _{2} 7$ & $\log _{2} 3$ \\
$S_{\mathrm{U}}\left(m_{\mathrm{i}}\right)$ & $\log _{2} 3$ & $\log _{2} 7$ & $2 \log _{2} 3$ \\
\hline
\end{tabular}

Table 2 The interval values of the proposed entropy of $m_{1}, m_{2}$ and $m_{3}$

\begin{tabular}{llll}
\hline Alternatives & $m_{1}$ & $m_{2}$ & $m_{3}$ \\
\hline$S\left(m_{i}\right)$ & {$\left[\log _{2} 3, \log _{2} 3\right]$} & {$\left[\log _{2} 7, \log _{2} 7\right]$} & {$\left[\log _{2} 3,2 \log _{2} 3\right]$} \\
\hline
\end{tabular}

Table 3 The golden rule method values of the proposed entropy of $m_{1}$, $m_{2}$ and $m_{3}$

\begin{tabular}{llll}
\hline Alternatives & $A_{1}$ & $A_{2}$ & $A_{3}$ \\
\hline $\operatorname{Rep}\left(A_{\mathrm{i}}\right)$ & $\log _{2} 3$ & $\log _{2} 7$ & $2 \log _{2} 3-1.5\left(\log _{2} 3\right)^{2}$ \\
\hline
\end{tabular}


Suppose that our uncertain evaluation of B is based on three attributes $Y=\left\{y_{1}, y_{2}, y_{3}\right\}$. Because Dempster-Shafer structure is a recognized tool that can efficiently represent unknown information, we use Dempster-Shafer structure to express this issue. According to the suggestions of experts, the Dempster-Shafer structure based on $\mathrm{Y}$ is used in this example, $m$, as follows:

$$
\begin{aligned}
& m\left(\left\{y_{1}\right\}\right)=0.2, m\left(\left\{y_{1}, y_{3}\right\}\right)=x, \\
& m\left(\left\{y_{1}, y_{2}, y_{3}\right\}\right)=0.8-x
\end{aligned}
$$

We use Tsallis entropy and the proposed entropy to evaluate this problem. The comparison curve of the two entropies is shown in Fig. 2.

In Fig. 2, when the non-extensive constant $q$ is increased in increments of 0.5 from 0.5 to 2.5 , the uncertainty of B calculated by Tsallis entropy is always less than the lower limit of the proposed entropy, which shows that the proposed entropy can obtain more information of Tsallis entropy. At the same time, because the uncertainty of an unknown information cannot be completely determined in practical applications, it is counterintuitive that Tsallis entropy obtains a certain uncertainty for an unknown object. The proposed entropy is an interval entropy model, and an interval of entropy can be obtained for an unknown information, which is also the reason why the proposed model is efficient.

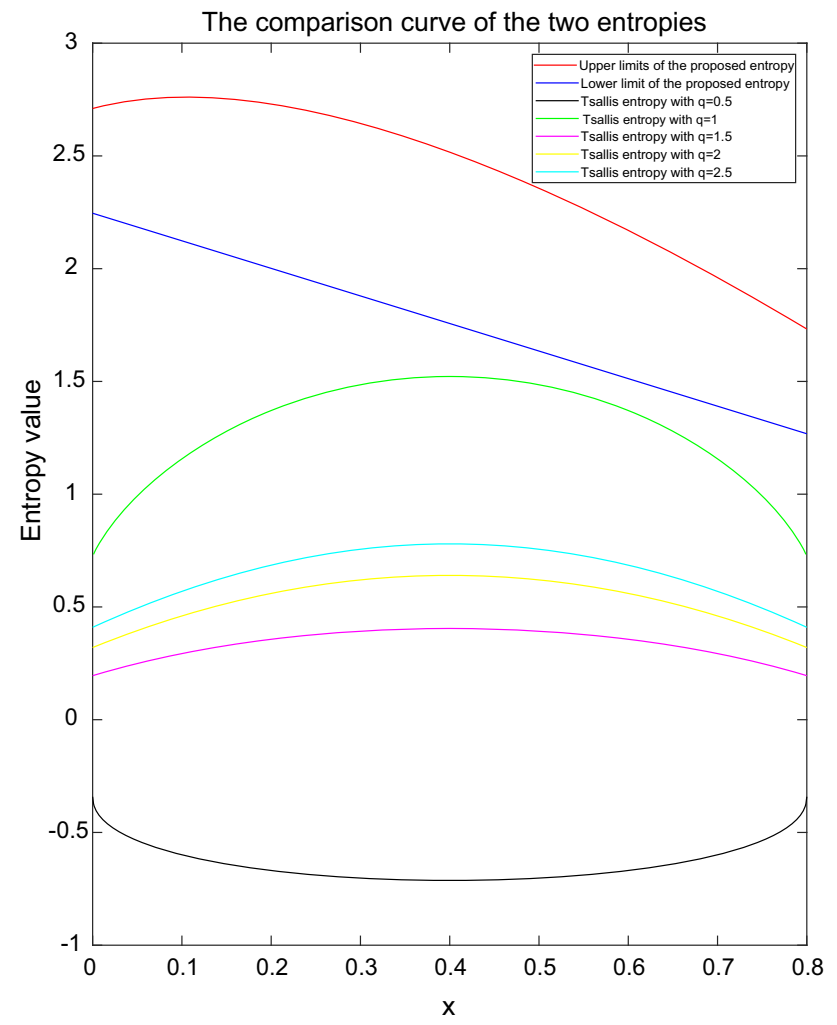

Fig. 2 The comparison curve of two entropies

\section{Conclusion}

This paper proposes interval-valued belief entropy for DempsterShafer structures, which is a new interval entropy model based on Dempster-Shafer structures and belief entropy. The proposed entropy can be based on the cardinality of the focal element in a given space and the belief and plausibility of the focal element of a given space to get an interval entropy value, which provides theoretical support for decision making. When belief entropy degenerates to classic information entropy, the proposed entropy degenerates to the intervalvalued entropies for Dempster-Shafer structures. Numerical examples and comparative experiment are used to verify the efficiency of the proposed entropy in decision making. The experimental results show that the proposed entropy can be better applied to solve practical problems than some other entropy models.

Quantum theory is currently the most popular and important theory. Dempster-Shafer evidence theory and quantum theory have similarities in many aspects, and belief entropy is the most efficient entropy to measure the uncertainty of unknown information in the evidence environment. Therefore, we will later improve the proposed model to adapt to the quantum environment. In other words, we will use the proposed entropy in future work to measure the uncertainty of the unknown information in the quantum system.

Acknowledgements The authors greatly appreciate the reviews' suggestions and the editor's encouragement. The work is partially supported by National Natural Science Foundation of China (Grant No. 61973332) and JSPS Invitational Fellowships for Research in Japan (Short-term).

\section{Declarations}

Conflict of interest All the authors certify that there is no conflict of interest with any individual or organization for the present work.

Animal and human rights This article does not contain any studies with human participants or animals performed by any of the authors.

\section{References}

Abellán J (2017) Analyzing properties of deng entropy in the theory of evidence. Chaos Solitons Fract 95:195-199

Abellan J, Bosse E (2018) Drawbacks of uncertainty measures based on the pignistic transformation. IEEE Trans Syst Man Cybern Syst 48(3):382-388

András T, Sándor BT, Zoltán N (2019) Entropic divergence and entropy related to nonlinear master equations. Entropy. https://doi.org/10. 3390/e21100993

Atanassov Krassimir T (1999) Intuitionistic fuzzy sets. Physica-Verlag HD, Berlin, pp 1-137

Baozhen D, Hongji X, Hailiang X, Zhengfeng D, Feifei L, Min C, Beibei Z, Qinghua X (2019) An improved ds evidence theory based on gray relational analysis. In: 2019 IEEE 4th International confer- 
ence on cloud computing and big data analysis (ICCCBDA), pages 640-644. IEEE

Biró TS, Néda Z, Telcs A (2019) Entropic divergence and entropy related to nonlinear master equations. Entropy 21(10):993

Deng Y (2020) Uncertainty measure in evidence theory. Sci China Inf Sci 63(11):210201

Deng J, Deng Y (2021) Information volume of fuzzy membership function. Int J Comput Commun Control 16(1):4106

Deng X, Jiang W (2019) A total uncertainty measure for D numbers based on belief intervals. Int J Intell Syst 34(12):3302-3316

Deng X, Jiang W (2019) Evaluating green supply chain management practices under fuzzy environment: a novel method based on D number theory. Int J Fuzzy Syst 21:1389-1402

Fan X, Guo Y, Ju Y, Bao J, Lyu W (2020) Multisensor fusion method based on the belief entropy and ds evidence theory. J Sensors. https://doi.org/10.1155/2020/7917512

Ferson S, Kreinovich V, Grinzburg L, Myers D, Sentz K (2015) Constructing probability boxes and dempster-shafer structures. Technical report, Sandia National Lab.(SNL-NM), Albuquerque, NM (United States)

Fu C, Xue M, Xu DL, Yang SLFuC, Xue M, Xu DL, Yang SL (2019) Selecting strategic partner for tax information systems based on weight learning with belief structures. Int J Approx Reason 105:66-84

Fuyuan X (2019) EFMCDM: evidential fuzzy multicriteria decision making based on belief entropy. IEEE Trans Fuzzy Syst. https:// doi.org/10.1109/TFUZZ.2019.2936368

Gend LP, Saha R (2019) Reeds: relevance and enhanced entropy based dempster shafer approach for next word prediction using language model. J Comput Sci 35:1-11

Huang Z, Yang L, Jiang W (2019) Uncertainty measurement with belief entropy on the interference effect in the quantum-like bayesian networks. Appl Math Comput 347:417-428

Huang J, Wang X, Wang D, Wang Z, Hua X (2019) Analysis of weak fault in hydraulic system based on multi-scale permutation entropy of fault-sensitive intrinsic mode function and deep belief network. Entropy 21(4):425

Hurley J, Johnson C, Dunham J, Simmons J (2019) Nonlinear algorithms for combining conflicting identification information in multisensor fusion. In: 2019 IEEE Aerospace Conference, pages 1-7. IEEE

Jaunzemis Andris D, Holzinger Marcus J, Chan Moses W, Shenoy Prakash P (2019) Evidence gathering for hypothesis resolution using judicial evidential reasoning. Inf Fusion 49:26-45

Jiang W, Huang C, Deng X (2019) A new probability transformation method based on a correlation coefficient of belief functions. Int $\mathrm{J}$ Intell Syst 34:1337-1347

Jirousek R, Shenoy PP (2018) A new definition of entropy of belief functions in the Dempster-Shafer theory. Int J Approx Reason 92:49-65

Kang B, Zhang P, Zhenyu Gao, Chhipi-Shrestha G, Hewage K, Sadiq R (2019) Environmental assessment under uncertainty using dempster-shafer theory and z-numbers. J Ambient Intell Hum Comput. https://doi.org/10.1007/s12652-019-01228-y

Karci Ali (2018) Notes on the published article "fractional order entropy: New perspectives" by ali karci, optik-international journal for light and electron optics, volume 127, issue 20, october 2016, pages 9172-9177. Optik 171:107-108

Kern-Isberner G, Rödder W (2004) Belief revision and information fusion on optimum entropy. Int J Intell Syst 19(9):837-857

Khan N, Anwar S (2019) Time-domain data fusion using weighted evidence and dempster-shafer combination rule: Application in object classification. Sensors 19(23):5187

Khoshtinat S, Aminnejad B, Hassanzadeh Y, Ahmadi H (2019) Groundwater potential assessment of the sero plain using bivariate models of the frequency ratio, shannon entropy and evidential belief function. J Earth Syst Sci 128(6):152
Kreinovich V, Kosheleva O, Sriboonchitta S (2021) How to reconcile maximum entropy approach with intuition: eg, should interval uncertainty be represented by a uniform distribution

Kuzemsky AL (2018) Temporal evolution, directionality of time and irreversibility. Rivista del Nuovo Cimento 41(10):513-574

Liu F, Gao X, Zhao J, Deng Y (2019) Generalized belief entropy and its application in identifying conflict evidence. IEEE Access 7(1):126625-126633

Michael B , Julio Urenda C , Olga K, Vladik K (06 2020) Which distributions (or families of distributions) best represent interval uncertainty: case of permutation-invariant criteria, pages 70-79

Mohd Jamal NJ, Ku Khalif KMN, Mohamad MS (2019) The implementation of z-numbers in fuzzy clustering algorithm for wellness of chronic kidney disease patients. J Phys Conf Ser 1366:012058

Moise Digrais M, Tchimou N, Nogbou Georges A, Souleymane O (2018) A new uncertainty measure in belief entropy framework. Power 9(11): 1

Moise DM, N'Takpe T, Nogbou GA, Oumtanaga S (2018) A new uncertainty measure in belief entropy framework. Int J Adv Comput Sci Appl 9(11):600-606

Ozkan K (2018) Comparing shannon entropy with deng entropy and improved deng entropy for measuring biodiversity when a priori data is not clear. J Fac For Istanbul Univ 68:136-140

Pan L, Deng Y (2020) Probability transform based on the ordered weighted averaging and entropy difference. Int J Comput Commun Control 15(4):3743

Pan Y, Zhang L, Li Z, Ding L (2019) Improved fuzzy bayesian networkbased risk analysis with interval-valued fuzzy sets and d-s evidence theory. IEEE Trans Fuzzy Syst 28:2063

Potyka N, Beierle C, Kern-Isberner G (2015) A concept for the evolution of relational probabilistic belief states and the computation of their changes under optimum entropy semantics. J Appl Logic 13(4):414-440

Seiti H, Hafezalkotob A (2018) Developing pessimistic-optimistic riskbased methods for multi-sensor fusion: an interval-valued evidence theory approach. Appl Soft Comput 72:609-623

Shafer G (1976) A mathematical theory of evidence, vol 42. Princeton University Press, Princeton

Tsallis C (1988) Possible generalization of boltzmann-gibbs statistics. J Stat Phys 52:479-487

Tuğal I, Karc1 A (2019) Comparisons of karc1 and shannon entropies and their effects on centrality of social networks. Phys A Stat Mech Appl 523:352-363

Turhan H, Demirekler M (2017) A novel combination methodology for dempster-shafer theory. In: 2017 25th Signal Processing and Communications Applications Conference (SIU), pages 1-4. IEEE

Vandoni J, Aldea E, Hégarat-Mascle SL (2019) Evidential query-bycommittee active learning for pedestrian detection in high-density crowds. Int J Approx Reason 104:166-184

Wang K (2018) Evidence combination method in time domain based on reliability and importance. J Syst Eng Electron 29(6):1308-1316

Wang Z, Xiao F (2019) An improved multi-source data fusion method based on the belief entropy and divergence measure. Entropy 21(6):611

Wang H, Fang YP, Zio E (2019) Risk assessment of an electrical power system considering the influence of traffic congestion on a hypothetical scenario of electrified transportation system in new york stat. IEEE Trans Intell Transp Syst. https://doi.org/10.1109/TITS. 2019.2955359

Wang J, Qiao K, Zhang Z (2019) An improvement for combination rule in evidence theory. Futur Gener Comput Syst 91:1-9

Wen J, Ying C, Xinyang D (2019) A Novel Z-network model based on bayesian network and Z-number. IEEE Trans Fuzzy Syst 28:1585

Xiao F (2019) A multiple-criteria decision-making method based on d numbers and belief entropy. Int J Fuzzy Syst 21(4):1144-1153 
Xing-Xian Zhang, Ying-Ming Wang, Sheng-Qun Chen, Lei Chen (2019) On the combination and normalization of conflicting interval-valued belief structures. Comput Ind Eng 137:

Xue Y, Deng Y, Garg H (2020) Uncertain database retrieval with measure-based belief function attribute values under intuitionistic fuzzy set. Inf Sci 546:436-447

Yager Ronald R (1999) A class of fuzzy measures generated from a dempster-shafer belief structure. Int J Intell Syst 14(12):12391247

Yager Ronald R (2017) Multi-criteria decision making with interval criteria satisfactions using the golden rule representative value. IEEE Trans Fuzzy Syst 26(2):1023-1031

Yager Ronald R (2018) Interval valued entropies for dempster-shafer structures. Knowledge-Based Syst 161:390-397

Yager Ronald R (2019) Extending set measures to pythagorean fuzzy sets. Int J Fuzzy Syst 21(2):343-354

Yager Ronald R, Reformat Marek Z, To Nhuan D (2019) Drawing on the ipad to input fuzzy sets with an application to linguistic data science. Inf Sci 479:277-291

Yingming Z, Hongji X, Junfeng S, Lingling P, Baozhen D, Min C (2018/04) Multisensor data fusion based on modified d-s evidence theory. In: 2018 international conference on computer modeling, simulation and algorithm (CMSA 2018). Atlantis Press
Yong Deng (2020) Information volume of mass function. Int J Comput Commun Control 15(6):3983

Zehong C, Chun-Hsiang C, Jung-Kai K, Chin-Teng L (2019) Multichannel EEG recordings during a sustained-attention driving task. Sci Data. https://doi.org/10.1038/s41597-019-0027-4

Zhang H, Deng Y (2021) Entropy measure for orderable sets. Inf Sci 561:141-151

Zhang Y, Liu Y, Zhang Z, Chao H-C, Zhang J, Liu Q (2017) A weighted evidence combination approach for target identification in wireless sensor networks. IEEE Access 5:21585-21596

Zhang X-X, Wang Y-M, Chen S-Q, Chu J-F (2019) Evidential reasoning rule for interval-valued belief structures combination. J Intell Fuzzy Syst 37(2):2231-2242

Publisher's Note Springer Nature remains neutral with regard to jurisdictional claims in published maps and institutional affiliations. 